\title{
CD4 count and tuberculosis risk in HIV-positive adults not on ART: A systematic review and meta-analysis
}

\author{
Penelope K Ellis ${ }^{1}$, Willam J Martin ${ }^{1}$ ， Peter J Dodd $^{\text {Corresp. } 2}$ \\ ${ }^{1}$ Sheffield Medical School, University of Sheffield, United Kingdom \\ 2 School of Health and Related Research, University of Sheffield, United Kingdom \\ Corresponding Author: Peter J Dodd \\ Email address: p.j.dodd@sheffield.ac.uk
}

Background : CD4 cell count in adults with human immunodeficiency virus (HIV) infection (PLHIV) not receiving antiretroviral therapy (ART) influences tuberculosis (TB) risk. Despite widespread use in models informing resource allocation, this relationship has not been systematically reviewed.

Methods : We systematically searched MEDLINE, Aids Info, Cochrane review database and Google Scholar for reports in English describing TB incidence stratified by updated CD4 cell count in cohorts of HIV-positive adults (age $>=15$ years) not on ART (PROSPERO protocol no: CRD42016048888). Among inclusion criteria were: reporting precision for TB incidence, repeated CD4 measurements, and TB incidence reported for those not on ART or monotherapy. Study quality was assessed via the NewcastleOttawa tool for cohort studies. A Bayesian hierarchical model was fitted to estimate the pooled factor increase in TB incidence with respect to CD4 cell count decrement.

Results : 1,555 distinct records were identified from which 164 full text articles were obtained. Common reasons for exclusion of full texts were: no valid TB incidence, no repeat CD4 measurements, and not reporting TB incidence by ART status. The 7 studies included reported on 1,206 TB cases among 41,271 individuals, with a typical median follow-up of 4 years. Studies were generally rated as moderate or high quality. Our meta-analysis estimated a 1.43 (95\% credible interval: 1.16 - 1.88)-fold increase in TB incidence per 100 cells per $\mathrm{mm}^{3}$ decrease in CD4 cell count.

Discussion : Our analysis confirms previous estimates of exponential increase in TB incidence with declining CD4 cell count in adults, emphasizing the importance of early ART initiation to reduce TB risk in PLHIV. 
1 CD4 count and tuberculosis risk in HIV-positive adults not on ART:

2 A systematic review and meta-analysis

3

\author{
Short title: CD4 count \& TB risk in PLHIV not on ART \\ P.K. ELLIS ${ }^{1, *}$, W.J. MARTIN ${ }^{1, *}$, and P.J. DODD, Ph.D ${ }^{2, \#}$ \\ 1. University of Sheffield Medical School, Sheffield, UK \\ 2. School of Health and Related Research, University of Sheffield, \\ Regent Court, 30 Regent Street, Sheffield, S1 4DA, UK \\ * joint first authors, contributed equally to this work \\ \# corresponding author, email: p.j.dodd@sheffield.ac.uk T: +441142220740 F: +441142220749
}

\begin{abstract}
Background: CD4 cell count in adults with human immunodeficiency virus (HIV) infection (PLHIV) not receiving antiretroviral therapy (ART) influences tuberculosis (TB) risk. Despite widespread use in models informing resource allocation, this relationship has not been systematically reviewed.

Methods: We systematically searched MEDLINE, Aids Info, Cochrane review database and Google Scholar for reports in English describing TB incidence stratified by updated CD4 cell count in cohorts of HIV-positive adults (age $>=15$ years) not on ART (PROSPERO protocol no: CRD42016048888). Among inclusion criteria were: reporting precision for TB incidence, repeated CD4 measurements, and TB incidence reported for those not on ART or monotherapy. Study quality was assessed via the Newcastle-Ottawa tool for cohort studies. A Bayesian hierarchical model was fitted to estimate the pooled factor increase in TB incidence with respect to $\mathrm{CD} 4$ cell count decrement.
\end{abstract}

Results: 1,555 distinct records were identified from which 164 full text articles were obtained. Common reasons for exclusion of full texts were: no valid TB incidence, no repeat CD4 measurements, and not reporting TB incidence by ART status. The 7 studies included reported on 1,206 TB cases among 41,271 individuals, with a typical median follow-up of 4 years. Studies were generally rated as moderate or high quality. Our meta-analysis estimated a 1.43 ( $95 \%$ credible interval: $1.16-1.88$ )-fold increase in TB incidence per 100 cells per $\mathrm{mm}^{3}$ decrease in CD4 cell count.

Discussion: Our analysis confirms previous estimates of exponential increase in TB incidence with declining CD4 cell count in adults, emphasizing the importance of early ART initiation to reduce TB risk in PLHIV.

\title{
Introduction
}


42 Tuberculosis (TB) and the human immunodeficiency virus (HIV) are the two leading infectious

43 killers with an estimated 1.4 million deaths from TB (excluding those with HIV) and 0.4 million

44 deaths in those with HIV and TB in 2015.(World Health Organization 2016) HIV is the most

45 potent known risk factor for TB disease incidence and has been a major factor in the upswing in

46 TB incidence in sub-Saharan Africa throughout the 1990s. Around 11\% of the 10.4 million be in people living with HIV (PLHIV) in 2015.(World Health Organization 2016) TB is implicated in $36 \%$ of deaths from HIV.(UNAIDS 2016)

Anti-retroviral therapy (ART) for in PLHIV is known to be around 70\% protective against TB incidence.(Suthar et al. 2012) Isoniazid preventive therapy (IPT) against TB is now recommended for 36 months in PLHIV, (World Health Organization 2015b) and further reduces the risk of TB by 30-50\%.(Golub et al. 2007; Rangaka et al. 2014; Samandari et al. 2011; Yirdaw et al. 2014) ART additionally has substantial benefits of reduced all-cause mortality.(Antiretroviral Therapy Cohort 2008) Despite encouraging recent progress however, under $50 \%$ of adults living with HIV are on ART,(UNAIDS 2016) and under $40 \%$ of PLHIV newly enrolled in care received IPT in 2015.(World Health Organization 2016)

$\mathrm{CD}^{+}$cells play a pivotal role in both HIV and Mycobacterium tuberculosis (M.tb) infection, and the peripheral blood $\mathrm{CD}^{+}$lymphocyte count (CD4 cell count) played an important role historically in ART initiation guidelines and has been shown to be a strong predictor of TB risk. Despite the fact that HIV treatment guidelines recommend ART initiation of regardless of CD4 count,(World Health Organization 2015a) individuals have typically undergone some CD4 decline by the time they are diagnosed and begin HIV treatment. Understanding the populationlevel implications of this increase in mean risk of TB is therefore still crucial to understanding the epidemiology of a given setting, predicting the impact of interventions to improve HIV diagnosis, and resource planning. Previous analyses (Williams \& Dye 2003; Williams et al. 2010) have found an exponential relationship between CD4 decrement in PLHIV not on ART and TB disease incidence, and their analysis has been widely used since in modeling analyses of the impact of HIV interventions on TB, linked HIV/TB resource planning (e.g. the OneHealth Tool frequently used to support applications to the Global Fund), and is used in TB burden estimation.(Pretorius et al. 2014a; Pretorius et al. 2014b; Williams \& Dye 2003; Williams et al. 2010) However, this relationship was not based on a transparent systematic review of available data.

Given the importance of the quantitative correspondence between CD4 count and TB risk in PLHIV not on ART and the absence of a systematic evidence synthesis, we sought to provide a meta-analytic estimate of this relation for adults based on systematically identified cohorts of ART-naïve PLHIV reporting TB incidence by updated CD4 count.

\section{Methods}

This systematic review is reported in accordance with the Preferred Reporting Items for Systematic Review and Meta-Analyses (PRISMA).(Moher et al. 2009) The protocol was 
87 registered with the International Prospective Register of Systematic Reviews (identification

88 number: CRD42016048888).

89

90

91

92

93

94

95

96

97

98

99

100

101

102

103

104

105

106

107

108

109

110

111

112

113

114

115

116

117

118

119

120

121

122

123

124

125

126

127

128

129

130

\section{1}

\section{Cohort definitions, selection criteria, and search strategy}

We sought cohorts of HIV-positive adults ( $>=15$ years of age) who were not on ART, with HIV infections and data stratified by CD4 count (exposure) at TB incidence (outcome). Studies were also included if they reported relative measures of TB incidence across CD4 categories, such as hazard ratios. The analysis comparison was between TB incidences for different CD4 count categories.

To be eligible, a study had to present empirical data on at least 5 cases of TB in adults. Studies were excluded if they explicitly restricted to groups not generally representative of adult TB cases in that population at that time, e.g. studies that only addressed one clinical manifestation of TB; that only comprised of hospitalized TB cases; studies that focused on migrant populations. Where explicit use of isoniazid preventive therapy was stated, those receiving IPT were excluded (similarly for any other form of prophylaxis). To allow appropriate study weighting, TB incidence or relative TB incidence had to be reported with confidence intervals, or as persontime and the number of incident TB cases.

To be eligible, studies had to report separately on individuals not on ART (ART including monotherapy etc.). Studies where the majority of HIV infections are known to be not HIV-1 were excluded. (HIV-1 is the most common major type of HIV.) We required at least one CD4 count measurement subsequent to the baseline measurement at enrolment.

If the same cohort was published more than once, or individuals were described in more than one cohort, studies were only included once.

We searched MEDLINE (11/16) for articles published in English at any time. (See Appendix for full search strategy.) The search was developed in consultation with an information specialist and sensitivity assessed by checking against known papers from Williams' analyses.(Williams \& Dye 2003; Williams et al. 2010) We also searched the AidsInfo database for registered studies that may have had relevant publications listed, and the Cochrane database of systematic reviews for relevant reviews.

The references of included studies were also searched for additional studies, as were articles listed as citing these articles on Google Scholar. 
132

133

134

135

136

137

138

139

140

141

142

143

144

145

146

147

148

149

150

151

152

153

154

155

156

157

158

159

160

161

\section{2}

163

164

165

166

167

168

169

170

171

172

173

174

175

176

Two reviewers (PKE \& WJM) independently screened articles returned by the search, and discrepancies were resolved in discussion with a third reviewer (PJD). Reasons for rejection were recorded.

A data extraction form was developed based on previous experience with a related review,(Dodd et al. 2017) and refined after application to the first included article. Data was independently extracted (by PKE \& WJM) on: first author, publication year, study country, study years, study description, ethnicity, CD4 cell count category, number in cohort, number of TB cases, crude and adjusted hazard ratios, patient years, TB incidence (and 95\% confidence interval[CI]), frequency of CD4 cell count measurement, proportion of TB bacteriologically confirmed, Bacille Calmette Guérin (BCG) vaccination coverage, tuberculin skin test (TST) status, age range, sex and HIV epidemic type. Any discrepancies were resolved by discussion with a third reviewed (PJD).

The 95\% Poisson exact confidence intervals based on reported event counts and person time were used to define precision for incidence data points. Where only adjusted hazard ratios with respect to a given CD4 category were reported, the stated $95 \%$ CIs were used to estimate precision. For the single TB incidence data point with zero TB events, we used the lower bound of the Poisson exact confidence interval divided by 100 as the central incidence estimate (as the logarithm of zero is not defined), and examined sensitivity to this approximation by repeating our analysis with an incidence point estimate value 10 times smaller again. CD4 category midpoints were used in statistical analyses with the assumption of an upper CD4 count of 1000 cells $/ \mathrm{mm}^{3}$ where not stated.

To assess risk of bias in individual studies, the Newcastle-Ottawa instrument for cohort studies(Wells et al. 2000) was adapted (see Appendix) and summarized as low-, moderate-, or high-quality for our question on selection, comparability and outcome domains depending on whether few, some, or most of the question items in a domain were answered positively.

\section{Statistical analysis}

In order to use information on both TB incidence and hazard ratios, we performed a Bayesian hierarchical meta-analysis assuming that the logarithmic TB incidence for a given CD4 category was proportional to a study-specific intercept (capturing the background TB incidence rate) and a study-specific gradient with respect to CD4 cell count drawn from a common distribution (analogously to a frequentist random-effects model). The model was fitted with Markov chain Monte Carlo (MCMC) methods in the R environment for statistical computing (R. Core Team) using RStan (Stan Development Team 2016) (see details and code in Appendix). Gelman-Rubin convergence statistics computed and chains plotted.

Heterogeneity was calculated as $\tau^{2}$, the variance of the summary distribution for the log-gradient, and a corresponding $I^{2}$ statistic evaluated using the summary statistic of (Higgins \& Thompson 2002) for individual studies' posterior variance. Forest plots of the individual and summary gradients with respect to CD4 were produced, and a funnel plot to assess risk of publication bias. 
177

178

179

180

181

182

183

184

185

186

187

188

189

190

191

192

193

194

195

196

197

198

199

200

201

202

203

204

205

206

207

208

209

210

211

212

213

214

215

216

217

218

219

220

221

We computed the implied factor increase in TB incidence per 100 cell per $\mathrm{mm}^{3}$ by our estimate, and similarly the incidence rate ratio for TB implied at different CD4 counts.

\section{Results}

See supporting PRISMA checklist for location of items reported.

\section{Study selection and characteristics}

Database searching returned 1,824 records, which together with 5 records identified by other means, led after screening to the full texts of 150 articles being examined (see flow diagram, Figure 1). The most common reason for exclusion at the full text stage was TB incidence not being reported (with an implied measure of precision); use of ART (or monotherapy) in the cohort with TB incidence measures without stratified reporting by ART status; and only CD4 at baseline being measured. The studies used in previous analyses (Williams \& Dye 2003; Williams et al. 2010) were captured by this strategy, but rejected for only measuring CD4 at baseline or not reporting TB incidence in PLHIV not on ART. Data from 7 studies was finally extracted for meta-analysis.

The 7 studies included are summarized in Table 1.(Assebe et al. 2015; Collins et al. 2015; Grant et al. 2009; Markowitz et al. 1997; Monge et al. 2014; Nicholas et al. 2011; Wolday et al. 2003) Studies included reported on 1,206 TB cases among 41,271 individuals, with a median over median follow-up times of 4.0 years. Three studies were based in sub-Saharan Africa (with heterosexual transmission predominating), one in Haiti, and the rest in the US or Europe (mostly men-who-have-sex with men, or injecting drug use), with most of the data being from the late 1990s or early 2000s. The more recent studies included evaluations of ART timing and IPT. The largest study was a retrospective observational cohort in the UK.(Grant et al. 2009) Less than half the studies reported ( 3 of 7) TST status, with the proportion of patients in cohorts being TST positive ranging between $6 \%$ and $38 \%$. The proportion of TB bacteriologically confirmed (reported by 4 of 7 studies) ranged between 50\% and 71\%. Only one study (Wolday et al. 2003) reported BCG vaccination status (38\% of PLHIV).

Most studies ( 5 of 7) reported person-time and incident TB cases by CD4 count; the two that did not (Assebe et al. 2015; Collins et al. 2015) both reported adjusted hazard ratios for CD4 categories derived from Cox proportional hazards models. In addition, around half of the incident TB cases reported in one study (Monge et al. 2014) were on ART, and so we used the adjusted hazard ratio for this study (which adjusted for ART status) in the meta-analysis. The studies analyzed as reporting TB incidence (4 of 7) contributed 868 incident TB cases during 50,045 person-years of observation. While the background TB incidence rates varied substantially between settings, the trends in TB incidence by CD4 count within study qualitatively supported the assumption of a linear relation to logarithmic TB incidence (see Figure 2). CD4 categories were not the same across studies, and typical intervals between measurements also varied. 
222

223

224

225

226

227

228

229

230

\section{1}

232

233

234

235

236

237

238

239

240

241

242

243

244

245

246

247

248

249

250

251

252

253

254

255

256

257

258

259

260

261

262

263

264

265

The studies were rated as having high comparability, since the comparison was between subgroups of the same cohort (see Table 1). Quality around outcome measurements was also generally good; likewise for selection of individuals (representativeness of PLHIV in given country, ascertainment of HIV status). The funnel plot (see Appendix) showed 3 of the 7 studies fell just outside the lower funnel boundary; formal tests of publication bias were not used since the number of studies included was fewer than 10 .

\section{Statistical analysis}

The Gelman-Rubin statistics for the MCMC chains (chains plotted in Appendix) were all $\leq 1.002$. The estimates of individual study log-TB incidence gradients with respect to CD4 are shown in the forest plot (see Figure 3 ) together with the summary estimate that was $0.36(95 \%$ credible interval [CrI]: $0.15-0.63$ ) per 100 cells $/ \mathrm{mm}^{3}$ (see Appendix for table). The heterogeneity measured by $\tau^{2}$ was $0.048(95 \% \mathrm{CrI}: 0.003-0.44) \operatorname{per}\left(100 \mathrm{cells} / \mathrm{mm}^{3}\right)^{2}$. This corresponds to an $I^{2}$ statistic of $96 \%$. The sensitivity analysis using a point-estimate of incidence a factor of 10 times smaller for the data point with no TB events yielded a log-TB incidence gradient with respect to CD4 of 0.40 (95\%CrI: $0.09-0.78)$.

This log-TB incidence gradient is equivalent to a 1.43 (95\% CrI: $1.16-1.88)$-fold increase in TB incidence risk per 100 cells per $\mathrm{mm}^{3}$ decline in CD4 count. The IRR implied by this result as CD4 cell-count declines rises from just under 3 for those with CD4 cell count over 500 cells per $\mathrm{mm}^{3}$ to over 25 for those with CD4 cell counts under 200 cells per $\mathrm{mm}^{3}$ (Figure 4).

\section{Discussion}

Our systematic review and meta-analysis confirms decline in CD4 cell count among HIVpositive adults (aged $>=15$ years) not receiving ART as a strong risk factor for incident TB, with the IRR for TB increasing exponentially as CD4 cell count declines. Our meta-analysis of the gradient of logarithmic TB incidence with respect to CD4 count is very close to the estimate produced previous analyses (Williams \& Dye 2003; Williams et al. 2010) but based on a nonoverlapping set of papers.

The papers included in previous analyses (Williams \& Dye 2003; Williams et al. 2010) were captured by our review but rejected on the grounds they did not separately report TB incidence off ART or only measured CD4 cell count at baseline (one of our a priori exclusion criteria). It is reassuring that our analysis reaches a very similar number from a different body of evidence, while including more patients from sub-Saharan Africa. However, in retrospect, since some of the studies we included had only infrequent CD4 measurement, e.g. (Collins et al. 2015) or did not report the regularity of measurement; it is not clear that their CD4 count categories are necessarily a more robust indicator of current CD4 count than a baseline measurement in study of short duration, e.g. (Antonucci et al. 1995) included in (Williams \& Dye 2003). Another limitation of our analysis is associated with CD4 cell count categories: these were broad and differed between studies, and for our meta-analyses we used category mid-point. 
We did not consider children (aged under 15 years) in our analysis, who have very different natural histories for both TB and HIV. The relationship between TB and HIV in children is the

269 subject of a separate systematic review and meta-analysis.(Dodd et al. 2017) It is possible that a

270

271

272

273

274

275

276

277

278

279

280

281

282

283

284

285

286

287

288

289

290

291

292

293

294

295

296

297

298

299

300

301

302

303

304

305

306

307

308

309

310 small number of individuals may have contributed person-time at age 14 to the cohort in (Monge et al. 2014).

A strength of this work is that the data included was based on the results of a systematic review with a clearly defined a priori search strategy, which captured all relevant articles of which we were aware. Our search did have limitations however: it restricted to articles in English; and while it included MEDLINE, it did not include another large general database such as Embase. The quality of studies included was generally rated as high for our analysis, although our assessment tool did not capture shortcomings such as the timing of CD4 measurements. There were too few studies included to formally assess evidence of publication bias.

The prevalence of Mycobacterium tuberculosis (M.tb) infection as measured by tuberculin skin test (TST) was available for a minority of studies. This is relevant because infection with M.tb is thought to confer protection against incident disease from re-infection in HIV-uninfected individuals,(Andrews et al. 2012) and it may be that HIV increases susceptibility to M.tb infection. The protection conferred by previous infection is often assumed to be absent in PLHIV, but quantitative evidence is lacking due to the particular problems of TST as a test for infection in this population.(Ayubi et al. 2016) Ultimately, this means the relationship analyzed is between CD4 count and TB incidence either from primary infection, re-activation or re-infection. HIV and CD4 decline may impact differently on the different routes to TB disease.

The statistical heterogeneity associated with our included studies was high. However with few studies and only study-level covariates usually available, we were not able to investigate potential causes of heterogeneity. These may have included differences between populations, strains of TB or HIV, by epidemic type or transmission route, and confounding by age or sex. BCG vaccination status was not reported, except for one study, (Wolday et al. 2003) and may itself have confounded results, either through variation in coverage or variation in efficacy.

Despite the shortcomings discussed above, confidence in our results in increased by the closeness to previous similar analyses, as well as with expectations from other studies not based on TB incidence in cohorts. The average predicted IRR over all CD4 counts $(\sim 8)$ is not dissimilar to the early population estimates (Corbett et al. 2003) based on the prevalence of HIV in TB cases. The increase in TB risk immediately following sero-conversion has been estimated as $\sim 2$, (Sonnenberg et al. 2005) which is compatible with the average of $\sim 2$ over the CD4 category 500-1000 cells per $\mathrm{mm}^{3}$.

It is noteworthy that many of the limitations we discuss above could be circumvented by an individual patient meta-analysis. This would obviate the CD4 category mid-point assumption and time since the last CD4 measurement could be used (potentially in conjunction with imputation via models of CD4 dynamics). The effects of potential confounders such as age, sex and HIV infection route could be considered. Other questions, such as the impact of incident TB disease

311 on CD4 dynamics or the relationship between updated CD4 cell count, time on ART, and TB 
312 incidence in PLHIV who are on ART might also be accessible. This last question, in addition to

313 its intrinsic interest, would also allow individual patient models to include more detail and

314 heterogeneity in their representations of TB risk.

\section{Conclusions}

The high risk of TB conferred by untreated HIV infection, the large fraction of HIV deaths associated with TB, and the still substantial numbers of PLHIV who are not on treatment underscores the importance of HIV testing, prompt ART initiation and TB-preventive therapy. Our analysis affirms the quantitative relationship underlying many TB models used in epidemic projection and resource planning. Our review and analysis both highlight the potential for an individual patient meta-analysis to improve our quantitative understanding of the relationship between HIV infection and TB risk.

\section{Figures titles \& legends}

Figure 1: PRISMA flow diagram of review process

Figure 2: Incidence of tuberculosis in adults living with HIV (age >=15 years) not on antiretroviral therapy, by study and CD4-positive lymphocyte count. Study data reported as incidence are plotted as triangles with confidence intervals; study data reported as hazard ratios are reported as dots, with confidence intervals except for the reference category. Note: the rightmost data point for Wolday, 2003 had zero TB cases which is not defined on a logarithmic scale - only the top of the Poisson exact confidence interval is shown, with the bottom truncated.

Figure 3: Forest plot of the rate of increase logarithmic tuberculosis incidence with CD4-positive lymphocyte count in adults living with HIV (age >=15 years) not on antiretroviral therapy. Summary and individual study measures are based on the posteriors from the hierarchical meta-analysis.

Figure 4: Increase in relative risk of tuberculosis incidence in adults living with HIV (age >=15 years) not on point estimate (thick solid line); horizontal lines represent means over depicted CD4 categories; dotted lines represent 95\% 
342

343

344

345

346

347

348

349

350

351

352

353

354

355

356

357

358

359

360

361

362

363

364

365

366

367

368

369

370

371

372

373

374

375

376

377

378

379

380

381

382

383

384

385

\section{References}

Andrews JR, Noubary F, Walensky RP, Cerda R, Losina E, and Horsburgh CR. 2012. Risk of progression to active tuberculosis following reinfection with Mycobacterium tuberculosis. Clin Infect Dis 54:784-791. 10.1093/cid/cir951

Antiretroviral Therapy Cohort C. 2008. Life expectancy of individuals on combination antiretroviral therapy in high-income countries: a collaborative analysis of 14 cohort studies. Lancet 372:293-299. 10.1016/S0140-6736(08)61113-7

Antonucci G, Girardi E, Raviglione MC, and Ippolito G. 1995. Risk factors for tuberculosis in HIV-infected persons. A prospective cohort study. The Gruppo Italiano di Studio Tubercolosi e AIDS (GISTA). JAMA 274:143-148.

Assebe LF, Reda HL, Wubeneh AD, Lerebo WT, and Lambert SM. 2015. The effect of isoniazid preventive therapy on incidence of tuberculosis among HIV-infected clients under preART care, Jimma, Ethiopia: a retrospective cohort study. BMC Public Health 15:346. 10.1186/s12889-015-1719-0

Ayubi E, Doosti-Irani A, Sanjari Moghaddam A, Sani M, Nazarzadeh M, and Mostafavi E. 2016. The Clinical Usefulness of Tuberculin Skin Test versus Interferon-Gamma Release Assays for Diagnosis of Latent Tuberculosis in HIV Patients: A Meta-Analysis. PLoS ONE 11:e0161983. 10.1371/journal.pone.0161983

Collins SE, Jean Juste MA, Koenig SP, Secours R, Ocheretina O, Bernard D, Riviere C, Calnan M, Dunning A, Hurtado Rua SM, Johnson WD, Jr., Pape JW, Fitzgerald DW, and Severe P. 2015. CD4 deficit and tuberculosis risk persist with delayed antiretroviral therapy: 5year data from CIPRA HT-001. Int J Tuberc Lung Dis 19:50-57. 10.5588/ijtld.14.0217

Corbett EL, Watt CJ, Walker N, Maher D, Williams BG, Raviglione MC, and Dye C. 2003. The growing burden of tuberculosis: global trends and interactions with the HIV epidemic. Arch Intern Med 163:1009-1021. 10.1001/archinte.163.9.1009

Dodd PJ, Prendergast AJ, Beecroft C, Kampmann B, and Seddon JA. 2017. The impact of HIV and antiretroviral therapy on TB risk in children: a systematic review and meta-analysis. Thorax. 10.1136/thoraxjnl-2016-209421

Golub JE, Saraceni V, Cavalcante SC, Pacheco AG, Moulton LH, King BS, Efron A, Moore RD, Chaisson RE, and Durovni B. 2007. The impact of antiretroviral therapy and isoniazid preventive therapy on tuberculosis incidence in HIV-infected patients in Rio de Janeiro, Brazil. AIDS 21:1441-1448. 10.1097/QAD.0b013e328216f441

Grant AD, Bansi L, Ainsworth J, Anderson J, Delpech V, Easterbrook P, Fisher M, Gazzard B, Gilson R, Gompels M, Hill T, Johnson M, Leen C, Orkin C, Phillips AN, Porter K, Post F, Walsh J, Sabin CA, and United Kingdom Collaborative HIVCSG. 2009. Tuberculosis among people with HIV infection in the United Kingdom: opportunities for prevention? AIDS 23:2507-2515. 10.1097/QAD.0b013e3283320dfd

Higgins JP, and Thompson SG. 2002. Quantifying heterogeneity in a meta-analysis. Stat Med 21:1539-1558. 10.1002/sim.1186

Markowitz, Hansen, Hopewell, Glassroth, Kvale, Mangura, Wilcosky, Wallace, Rosen, and Reichman. 1997. Incidence of tuberculosis in the United States among HIV-infected persons. The Pulmonary Complications of HIV Infection Study Group. Annals of Internal Medicine 126(2):123-32, 1997 Jan 15. 
386

387

388

389

390

391

392

393

394

395

396

397

398

399

400

401

402

403

404

405

406

407

408

409

410

411

412

413

414

415

416

417

418

419

420

421

422

423

424

425

426

427

428

429

430

Moher D, Liberati A, Tetzlaff J, and Altman DG. 2009. Preferred reporting items for systematic reviews and meta-analyses: the PRISMA statement. Annals of Internal Medicine 151:264-269.

Monge S, Diez M, Pulido F, Iribarren JA, Campins AA, Arazo P, Montero M, Miro JM, Moreno S, Del Amo J, and Spanish ARNC. 2014. Tuberculosis in a cohort of HIV-positive patients: epidemiology, clinical practice and treatment outcomes. Int J Tuberc Lung Dis 18:700-708. 10.5588/ijtld.13.0778

Nicholas, Sabapathy, Ferreyra, Varaine, Pujades R, and Sans AWGoM. 2011. Incidence of tuberculosis in HIV-infected patients before and after starting combined antiretroviral therapy in 8 sub-Saharan African HIV programs. Journal of Acquired Immune Deficiency Syndromes: JAIDS 57(4):311-8, 2011 Aug 1.

Pretorius C, Glaziou P, Dodd PJ, White R, and Houben R. 2014a. Using the TIME model in Spectrum to estimate tuberculosis-HIV incidence and mortality. AIDS 28 Suppl 4:S477487. 10.1097/QAD.0000000000000484

Pretorius C, Menzies NA, Chindelevitch L, Cohen T, Cori A, Eaton JW, Fraser C, Gopalappa C, Hallett TB, Salomon JA, Stover J, White RG, and Dodd PJ. 2014b. The potential effects of changing HIV treatment policy on tuberculosis outcomes in South Africa: results from three tuberculosis-HIV transmission models. AIDS 28 Suppl 1:S25-34. 10.1097/QAD.0000000000000085

R. Core Team. R: A language and environment for statistical computing. $R$ Foundation for Statistical Computing, Vienna, Austria. URL http://www.R-project.org.

Rangaka MX, Wilkinson RJ, Boulle A, Glynn JR, Fielding K, van Cutsem G, Wilkinson KA, Goliath R, Mathee S, Goemaere E, and Maartens G. 2014. Isoniazid plus antiretroviral therapy to prevent tuberculosis: a randomised double-blind, placebo-controlled trial. Lancet 384:682-690. 10.1016/S0140-6736(14)60162-8

Samandari T, Agizew TB, Nyirenda S, Tedla Z, Sibanda T, Shang N, Mosimaneotsile B, Motsamai OI, Bozeman L, Davis MK, Talbot EA, Moeti TL, Moffat HJ, Kilmarx PH, Castro KG, and Wells CD. 2011. 6-month versus 36-month isoniazid preventive treatment for tuberculosis in adults with HIV infection in Botswana: a randomised, double-blind, placebo-controlled trial. Lancet 377:1588-1598. 10.1016/S01406736(11)60204-3

Sonnenberg P, Glynn JR, Fielding K, Murray J, Godfrey-Faussett P, and Shearer S. 2005. How soon after infection with HIV does the risk of tuberculosis start to increase? A retrospective cohort study in South African gold miners. J Infect Dis 191:150-158. $10.1086 / 426827$

Stan Development Team. 2016. RStan: the $R$ interface to Stan. $R$ package version 2.14.1 URL http://mc-stan.org

Suthar AB, Lawn SD, del Amo J, Getahun H, Dye C, Sculier D, Sterling TR, Chaisson RE, Williams BG, Harries AD, and Granich RM. 2012. Antiretroviral therapy for prevention of tuberculosis in adults with HIV: a systematic review and meta-analysis. PLoS Medicine 9:e1001270. 10.1371/journal.pmed.1001270

UNAIDS. 2016. Global AIDS update 2016. Geneva.

Wells GA, Shea B, O'connell D, Peterson JEA, Welch V, Losos M, Tugwell P, and others. 2000. The Newcastle-Ottawa Scale (NOS) for assessing the quality of nonrandomised studies in meta-analyses. 
431 Williams BG, and Dye C. 2003. Antiretroviral drugs for tuberculosis control in the era of HIV/AIDS. Science 301:1535-1537. 10.1126/science.1086845 therapy for tuberculosis control in nine African countries. Proc Natl Acad Sci U S A 107:19485-19489. 10.1073/pnas.1005660107

Wolday, Hailu, Girma, Hailu, Sanders, and Fontanet. 2003. Low CD4+ T-cell count and high HIV viral load precede the development of tuberculosis disease in a cohort of HIVpositive Ethiopians. International Journal of Tuberculosis \& Lung Disease 7(2):110-6, $2003 \mathrm{Feb}$.

World Health Organization. 2015a. Guideline on when to start antiretroviral therapy and on preexposure prophylaxis for HIV. Geneva.

World Health Organization. 2015b. Recommendation on 36 months isoniazid preventive therapy to adults and adolescents living with HIV in resource-constrained and high TB-and HIVprevalence settings - 2015 update. Geneva.

World Health Organization. 2016. Global tuberculosis report 2016. Geneva.

Yirdaw KD, Jerene D, Gashu Z, Edginton ME, Kumar AM, Letamo Y, Feleke B, Teklu AM, Zewdu S, Weiss B, and Ruff A. 2014. Beneficial effect of isoniazid preventive therapy and antiretroviral therapy on the incidence of tuberculosis in people living with HIV in Ethiopia. PLoS ONE 9:e104557. 10.1371/journal.pone.0104557 
Figure 1 (on next page)

PRISMA flow diagram of review process 
Records identifiecthrough database searching $(n=1824)$

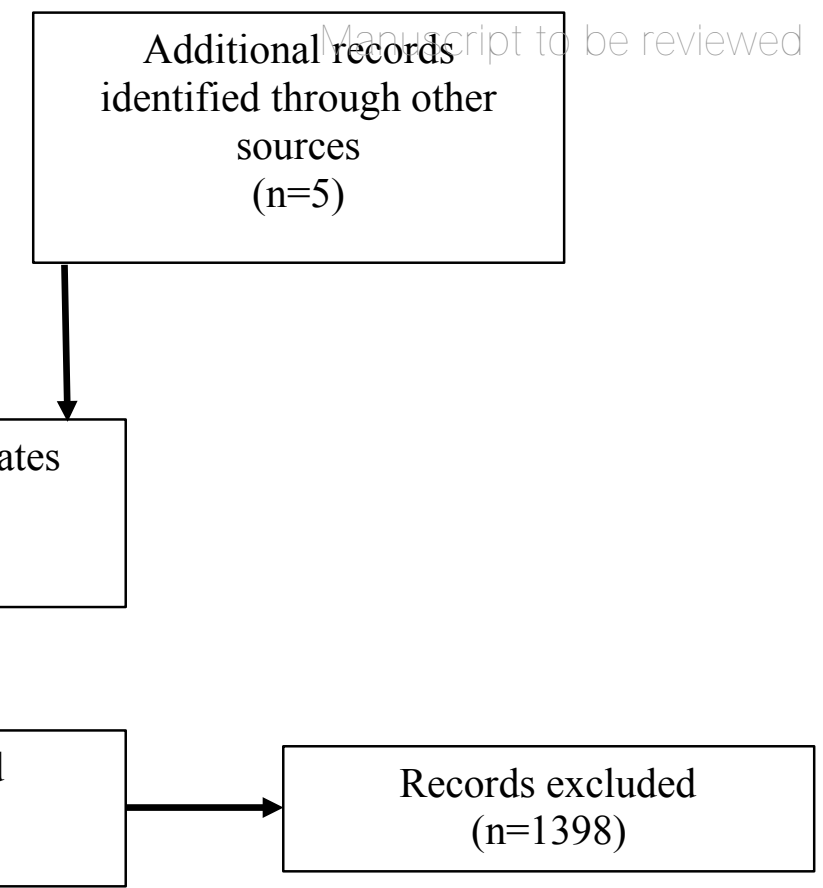

Full text articles excluded

$(n=150)$

Hospitalised population (7)

No measure of incidence (69)

No. of study cases $<5$ (1)

Article is a review (1)

Not representative of general population (3)

Only baseline CD4 taken (18)

Only representative of one specific type of TB (6)

Patient population aged $<15$ years (1)

Patients taking IPT or other forms of prophylaxis (1)

Patients on ART (including monotherapy) (17)

TB incidence not reported by CD4 cell count (10)

TB not primary outcome (3)

TB prevalent at beginning of study (13) 
Figure 2 (on next page)

Incidence of tuberculosis in adults living with HIV (age $>=15$ years) not on antiretroviral therapy, by study and CD4-positive lymphocyte count.

Study data reported as incidence are plotted as triangles with confidence intervals; study data reported as hazard ratios are reported as dots, with confidence intervals except for the reference category. Note: the rightmost data point for Wolday, 2003 had zero TB cases which is not defined on a logarithmic scale - only the top of the Poisson exact confidence interval is shown, with the bottom truncated. 


\section{Figure 3 (on next page)}

Forest plot of the rate of increase logarithmic tuberculosis incidence with CD4-positive lymphocyte count in adults living with HIV (age $>=15$ years) not on antiretroviral therapy.

Summary and individual study measures are based on the posteriors from the hierarchical meta-analysis. 


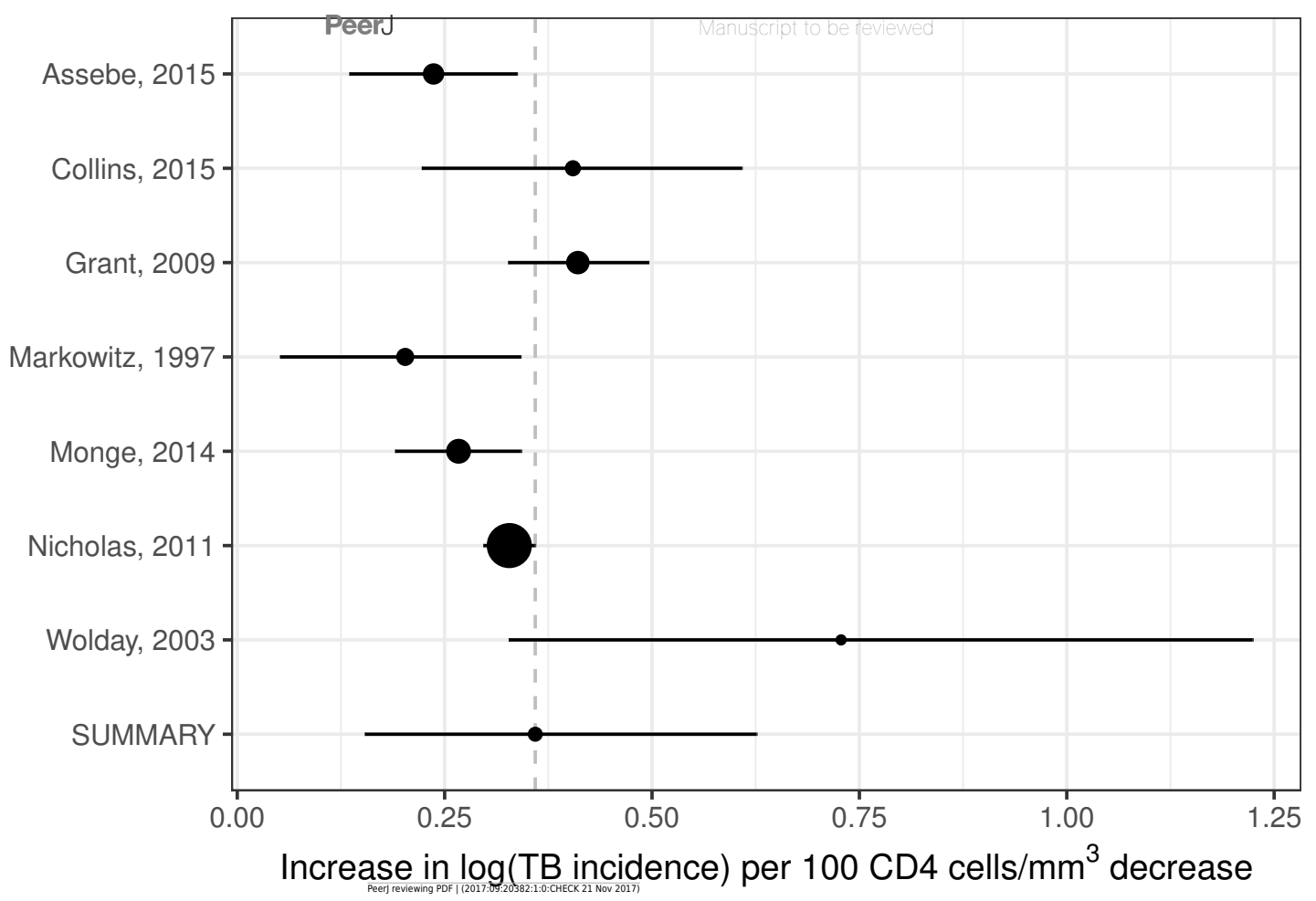




\section{Figure 4 (on next page)}

Increase in relative risk of tuberculosis incidence in adults living with HIV (age > =15 years) not on antiretroviral therapy by CD4-positive lymphocyte count.

Thick dashed lines represent 95\% credible intervals around the point estimate (thick solid line); horizontal lines represent means over depicted CD4 categories; dotted lines represent 95\% credible intervals for these category means; the horizontal dashed line represents an incidence rate ratio of 1 (no change). It is assumed that individuals have a CD4 count of 1000 cells $/ \mathrm{mm}^{3}$ at the point of HIV infection. 


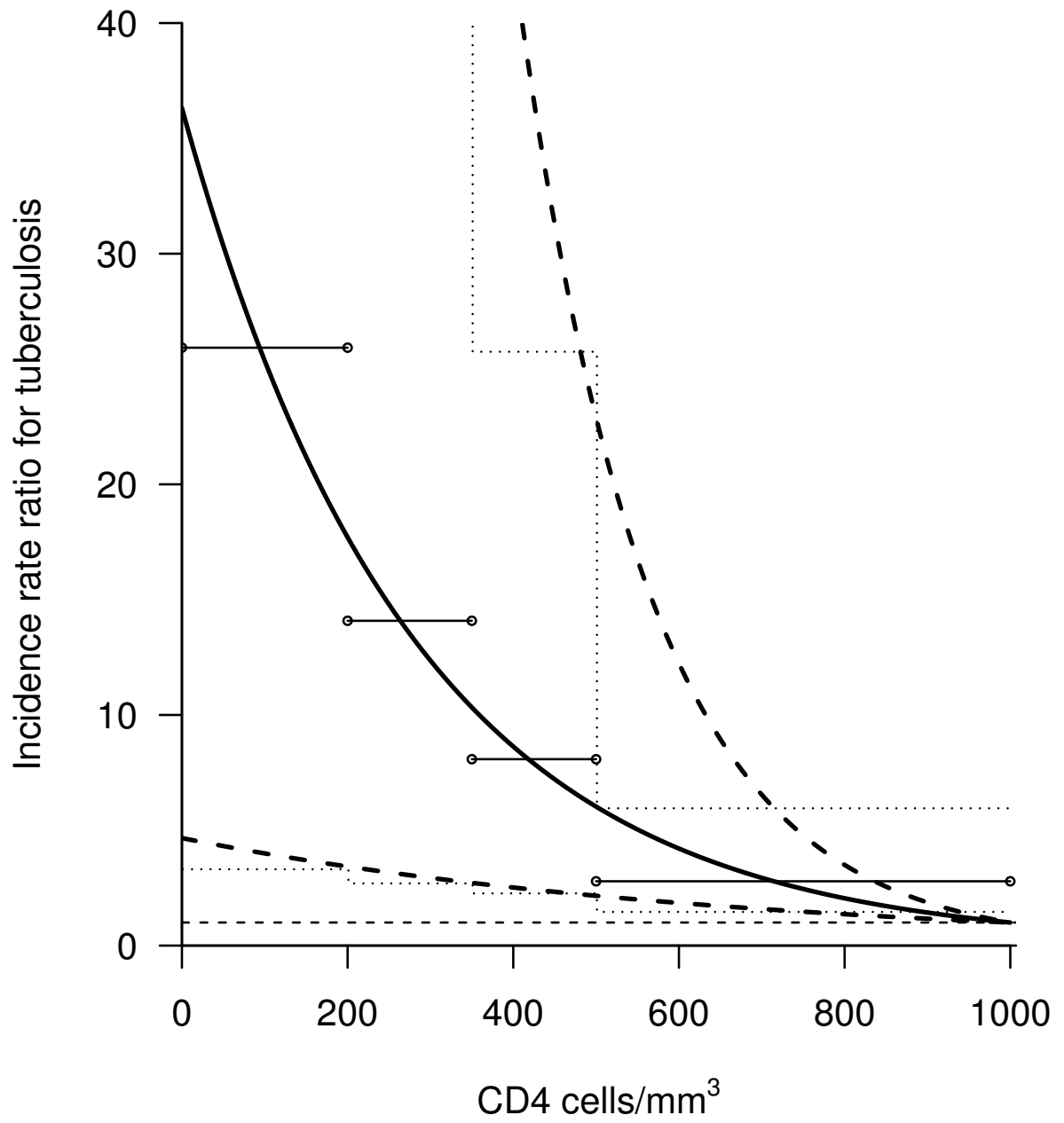




\section{Table $\mathbf{1}$ (on next page)}

Summary of studies 
1 Summary of studies.

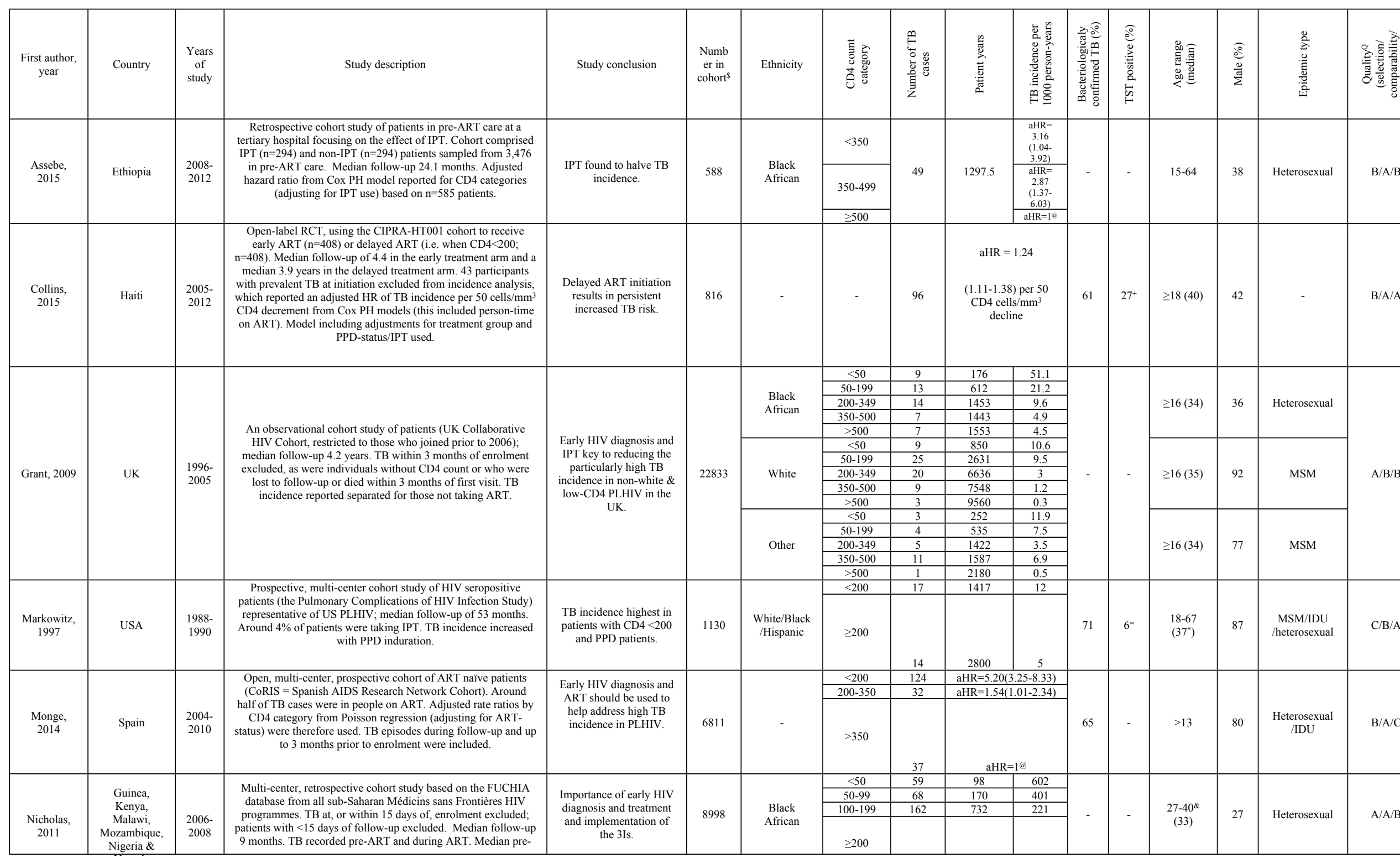




\begin{tabular}{|c|c|c|c|c|c|c|c|c|c|c|c|c|c|c|c|}
\hline & & & ART follow-up of 9 months. & & & & & & & & & & & & \\
\hline \multirow[b]{2}{*}{$\begin{array}{l}\text { Wolday, } \\
2003\end{array}$} & \multirow[b]{2}{*}{ Ethiopia } & \multirow[b]{2}{*}{$\begin{array}{l}1997- \\
2001\end{array}$} & \multirow{2}{*}{$\begin{array}{l}\text { A prospective cohort study (Ethio-Netherlands AIDS Research } \\
\text { Project=ENARP) to study biomarkers associated with TB } \\
\text { progression. HIV-positive and negative factory workers with an } \\
\text { overall median follow-up of } 3.8 \text { years and } 6 \text {-monthly } \\
\text { assessments. }\end{array}$} & \multirow{2}{*}{$\begin{array}{l}\text { Low CD4 and high viral } \\
\text { load associated with TB } \\
\text { incidence; successful TB } \\
\text { treatment does not reduce } \\
\text { viral load. }\end{array}$} & \multirow[b]{2}{*}{95} & \multirow[b]{2}{*}{$\begin{array}{l}\text { Black } \\
\text { African }\end{array}$} & $\begin{array}{c}<200 \\
200-499\end{array}$ & $\frac{5}{5}$ & $\begin{array}{l}46 \\
121\end{array}$ & $\frac{107.6}{41.2}$ & \multirow[b]{2}{*}{50} & \multirow[b]{2}{*}{$38^{=}$} & \multirow[b]{2}{*}{ (34) } & \multirow[b]{2}{*}{52} & \multirow[b]{2}{*}{ Heterosexual } \\
\hline & & & & & & & $\geq 500$ & 0 & 43 & 0 & & & & & \\
\hline
\end{tabular}

$(\mathrm{Q}=$ quality assessed with a modified Newcastle-Ottawa scale for cohorts with $\mathrm{A} / \mathrm{B} / \mathrm{C}$ according to all/some/few criteria met in each domain; ${ }^{*}=$ mean; ${ }^{\&}=$ inter-quartile range; ${ }^{=}=5 \mathrm{~mm}$ cut-off used in tuberculin skin test; ${ }^{+}=$cut-off used in tuberculin skin test not stated; ${ }^{\circledR}=$ reference value; $\$=$ cohort number restricted to those included in TB incidence calculation; aHR $=$ adjusted hazard ratio; $\mathrm{ART}=$ antiretroviral therapy; IPT $=$ isoniazid preventive therapy; $\mathrm{TB}=$ tuberculosis; $\mathrm{RCT}=$ randomized controlled trial; PPD = purified protein derivative; TST = tuberculin skin test; PLHIV = people living with HIV; IDU = injecting drug users; MSM = men who have sex with men) 